\title{
Eficiencia energética de colectores solares con tubos verticales de PVC en termas solares, Amazonas
}

\section{Energy efficiency of solar collectors with PVC vertical tubes in solar thermal systems, Amazonas}

\author{
Fernando Isaac Espinoza Canaza ${ }^{1 *}$ (iD) y Miguel Ángel Barrena Gurbillón ${ }^{1}(\mathbb{D}$
}

\section{RESUMEN}

La investigación tuvo como objetivo determinar la eficiencia de un colector solar de $1 \mathrm{~m}^{2}$ de 12 tubos verticales de PCV de 3/4" para agua caliente, con tanque de almacenamiento de 100 litros, en Chachapoyas, Amazonas (2350 msnm y temperatura ambiente promedio de $17^{\circ} \mathrm{C}$ ), con humedad relativa entre $60 \%$ y $70 \%$. Para la medición y registro de datos de temperatura se utilizaron cuatro sensores DS18B20 digitales y un módulo arduino UNO. Los datos se tomaron durante 31 días, en intervalos de 15 minutos, desde las 8:00 a las 16:00 horas en simultáneo con el registro de radiación solar, temperatura ambiente, precipitación pluvial y velocidad del viento con la estación meteorológica del INDES-CES. En base a los valores de radiación solar incidente. Cada día se clasificó en tres intervalos: nublados $\left(0\right.$ a $\left.300 \mathrm{~W} / \mathrm{m}^{2}\right)$, combinados $\left(600\right.$ a $\left.800 \mathrm{~W} / \mathrm{m}^{2}\right)$ y soleados (mayores a $\left.800 \mathrm{~W} / \mathrm{m}^{2}\right)$. La radiación solar captada por el colector solar en periodos nublados fue de $1,90 \mathrm{~kW} / \mathrm{m}^{2}$, combinados $0,89 \mathrm{~kW} / \mathrm{m}^{2}$, y soleados 2,04 $\mathrm{kW} / \mathrm{m}^{2}$. Por otra parte la eficiencia lograda para períodos nublados fue de $35,64 \%$, combinados $38,51 \%$, y soleados $44,57 \%$. El almacenamiento de agua en el tanque para periodos nublados fue de 87,10 litros, combinados 89,52 litros, y soleados 90,51 litros. En conclusión, la eficiencia del colector solar estuvo entre el 30 y $50 \%$.

Palabras claves: energías renovables, energía solar, medio ambiente, radiación solar.

\begin{abstract}
The objective of the research was to determine the efficiency of a $1 \mathrm{~m}^{2}$ solar collector with 12 vertical $3 / 4$ " PVC tubes for hot water, with a 100 litres storage tank, in Chachapoyas, Amazonas (2350 meters above sea level and average ambient temperature of $17^{\circ} \mathrm{C}$ ), with relative humidity between $60 \%$ and $70 \%$. For the measurement and recording of temperature data, four digital DS18B20 sensors and one arduino UNO module were used. The data was taken during 31 days, in 15 minute intervals, from the 8:00 to 16:00 hours simultaneous with the solar radiation register, coinciding with the meteorological station report INDES-CES that provided data on solar radiation, ambient temperature, precipitation and wind speed. Based on the level of solar radiation incident in one day, it was classified into three intervals: cloudy $\left(0\right.$ to $\left.300 \mathrm{~W} / \mathrm{m}^{2}\right)$, combined $\left(600\right.$ to $\left.800 \mathrm{~W} / \mathrm{m}^{2}\right)$ and sunny (over $\left.800 \mathrm{~W} / \mathrm{m}^{2}\right)$. The solar radiation collected by the solar collector in cloudy periods was $1.90 \mathrm{~kW} / \mathrm{m}^{2}$, combined $0.89 \mathrm{~kW} / \mathrm{m}^{2}$ and sunny 2.04 $\mathrm{kW} / \mathrm{m}^{2}$, while that the efficiency achieved for cloudy periods was $35.64 \%$, combined $38.51 \%$ and sunny $44.57 \%$. The storage of water in the tank for cloudy periods was 87.10 liters, combined 89.52 liters and sunny 90.51 liters. In conclusion, the efficiency of the solar collector is between 30 and $50 \%$.
\end{abstract}

Keywords: renewable energies, solar energy, environment, solar radiation.

\footnotetext{
${ }^{1} 1$ Universidad Nacional Toribio Rodríguez de Mendoza de Amazonas (UNTRM-A), Calle Higos Urco N 342-350-356, Calle Universitaria N ${ }^{\circ}$ 304, Chachapoyas, Perú

*Autor de Correspondencia, e-mail fernando.espinoza@untrm.edu.pe
} 


\section{INTRODUCCIÓN}

La energía solar es una fuente de energía renovable que se usa desde la antigüedad con diferentes fines. El calentamiento de agua empleando energía solar es un método común en varios países del mundo. Eeen algunos casos, una terma solar es usada como suplemento de un calentador convencional de agua, con el que se puede ahorrar hasta el 90\% de energía. En ciertas partes del planeta no es necesario un calentador convencional de agua, pues la terma solar puede abastecer suficiente agua caliente $\left(38^{\circ} \mathrm{C}\right.$ a $\left.55^{\circ} \mathrm{C}\right)$ (Paz Gutiérrez, 2006).

Las tecnologías desarrolladas para convertir la energía solar en energía utilizable por el hombre dependen de la disponibilidad de la radiación solar, que varía de manera importante en diferentes climas y regiones. Para seleccionar y diseñar la tecnología más apropiada para una región en particular, es necesario caracterizar la radiación solar en esa región. Datos confiables de radiación directa normal y radiación global son necesarios para el dimensionamiento de los sistemas de energía solar, así como para el estudio comparativo de los sitios de ubicación de los sistemas. Estos datos de radiación solar deberán estar disponibles junto con otros parámetros meteorológicos, como temperatura ambiente, temperatura de bulbo húmedo y velocidad del viento, que pueden afectar el comportamiento de algunos tipos de sistemas de energía solar (EstradaCajigal y Almanza, 2005).

En los últimos años, el estudio de la energía termo solar ha sido de gran interés debido a la crisis energética actual causada por el uso no adecuado de fuentes fósiles, principalmente en la generación de electricidad, que influye negativamente en el calentamiento global, en el deterioro de la capa de ozono, en la presencia de lluvias ácidas que traen como consecuencia la degradación del ambiente y de la calidad de vida (Yadav y Bajpai, 2011).

En el año 2006, se instalaron $3085265 \mathrm{~m}^{2}$ de colectores solares térmicos en Europa, equivalente a 2160 MW, que es $44 \%$ superior al año anterior, explicado por la crisis energética en Europa y por la voluntad política de algunos países para desarrollar el sector. Los colectores planos con cubierta son el componente principal de los equipos que se emplean para aprovechar la energía solar térmica en el mercado europeo, representando el 88,5\%, los colectores de tubos de vacío representan el 8,3\%, mientras que los colectores sin cubierta, sólo poseen el 3,2\% del mercado. Aproximadamente un 5\% de los hogares alemanes utilizan energía solar térmica. (INENCO, 2007).

La fabricación local de calentadores solares de agua es la tecnología más antigua y de mayor desarrollo y diseminación en el Perú. Se estima que hay 30000 termas solares, principalmente en Arequipa. Existen alrededor de 20 fabricantes que han constituido la “Asociación de Empresas Peruanas de Energía Solar" - AEPES, y que producen mensualmente alrededor de $600 \mathrm{~m}^{2}$ de colectores solares para termas solares (Horn, 2006). Esto demuestra que se está tomando conciencia de la necesidad de uso de la energía solar como alternativa para ahorrar recursos económicos y disminuir la contaminación ambiental.

La terma solar permite calentar agua con la radiación solar y almacenarla para ser usada en cualquier momento. La eficiencia y vida media de un calentador solar depende de los materiales y el diseño empleados. Consta de un colector solar, depósito de almacenamiento de agua y sistema de tuberías para conexión de agua fría y agua caliente. El colector solar puede utilizar la radiación solar difusa (reflejada por las nubes), además de la directa. El calentamiento del agua en el colector y su paso al tanque de almacenamiento tiene lugar entre las 9 y las 15 horas. Después, la temperatura del agua en el colector es menor que en la parte superior del tanque lo que interrumpe el termosifón, permaneciendo el agua caliente en el tanque, cuyo calor se conservará dependiendo de su aislamiento térmico (Valera, 2007).

El colector capta la energía solar y la transfiere al agua. El colector solar plano consiste en una superficie metálica que absorbe la radiación solar, sobre la que se adhiere la parrilla de tubos por los que circulará el agua que será calentada. Se coloca dentro de una estructura 
protectora (caja de madera o de plancha galvanizada), cuya parte superior se tapa con una lámina de vidrio y en su parte inferior y laterales se coloca material aislante (Fasulo et al., 2007).

Un colector solar de 1,8 a 2,1 $\mathrm{m}^{2}$ de superficie, conectado a un tanque de almacenamiento de 115 a 200 litros, aislado térmicamente, puede proporcionar agua a una temperatura de hasta $65^{\circ} \mathrm{C}$ en día soleado, en el que bastarán dos horas de exposición solar para tener agua caliente. Para obtener el $100 \%$ de la capacidad instalada, se necesitan de cinco a seis horas de insolación, dependiendo de la capacidad y del modelo (Pilatowsky, 2005).

El agua abastecida a los hogares en la ciudad de Chachapoyas y en muchas poblaciones urbanas y rurales dispersas ubicadas a más de 2000 msnm, en la Región Amazonas, se encuentra a una temperatura media anual de $14,5^{\circ} \mathrm{C}$ (Enperu, 2015), por lo que su empleo directo para el aseo personal puede causar enfermedades respiratorias, siendo los más expuestos los niños y los adultos mayores. Asimismo, la falta de aseo e higiene personal y familiar puede ocasionar afecciones a la piel y otras enfermedades.

En la zona urbana, para calentar el agua para el aseo personal se emplea energía convencional: terma eléctrica o gas propano en la cocina. Por el contrario, en las zonas rurales emplean leña. En ambos casos se incrementa el gasto económico mensual en los hogares. Una alternativa de solución para la cobertura de estas necesidades y que a la vez es económica, es la aplicación de la energía solar para calentar el agua.

En la Región Amazonas, no se cuenta con datos referenciales sobre el aprovechamiento de la energía solar para colectores solares. Sin embargo, se ha observado que en los últimos años, los hoteles y algunas viviendas urbanas en la ciudad de Chachapoyas son los que están optando por esta tecnología que no sobrepasan en su totalidad los $100 \mathrm{~m}^{2}$ en el uso de colectores solares. Por consiguiente, se puede afirmar que en la Región Amazonas hay un gran potencial para el desarrollo e implementación de los colectores solares para calentar agua.

\section{MATERIAY MÉTODOS}

La evaluación del colector solar de tubos verticales de PVC se realizó en la Estación Experimental Chachapoyas, en el Campus de la Universidad Nacional Toribio Rodríguez de Mendoza de Amazonas, entre los

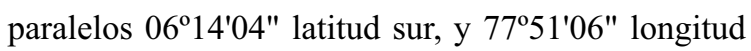
oeste, a una altitud de 2350 m.s.n.m., con temperatura ambiente promedio de $14,5^{\circ} \mathrm{C}$, en la ciudad de Chachapoyas, capital de la Región Amazonas.

\section{Metodología}

En la presente investigación se determinó la eficiencia del colector solar de CPVC, en función de la radiación solar con periodos nublados de $0-600 \mathrm{~W} / \mathrm{m}^{2}$, combinados entre 600 a $800 \mathrm{~W} / \mathrm{m}^{2}$, y soleados cuando sean mayores a $800 \mathrm{~W} / \mathrm{m}^{2}$.

\section{Material para hacer el colector solar}

El colector solar se construyó con un armazón de tubo de aluminio de 1,5" x 3,5" de color negro (Figura 1 y

2), cuyas características principales son:

- Área del colector solar: $1 \mathrm{~m}^{2}$

- Largo del interior del colector: 1,20 m

- Ancho del interior del colector: $0,84 \mathrm{~m}$

- Largo total del colector: 1,28 m

- Ancho total del colector: 0,92 m

- Profundidad del colector: $0,089 \mathrm{~m}$

- Conductos del colector: Tubos PVC de 3/4"

- Cantidad de conductos de PVC: 12

- Tapa del colector: vidrio incoloro de $4 \mathrm{~mm}$

- Aislante de colector: Tecnopor de 2"

- Absorbente de radiación solar: calamina de $0,5 \mathrm{~mm}$

- Tipo de pintura esmalte: Negro mate.

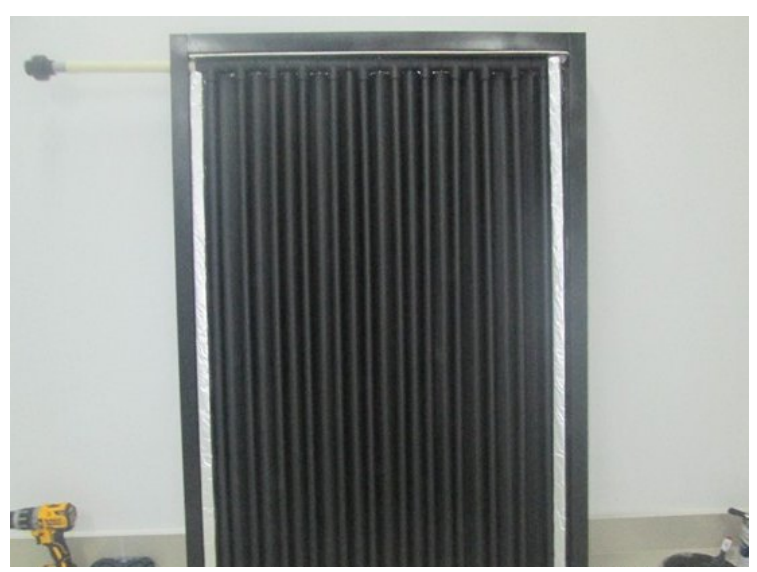

Figura 1. Colector solar con tubos de 3/4" de PVC. 


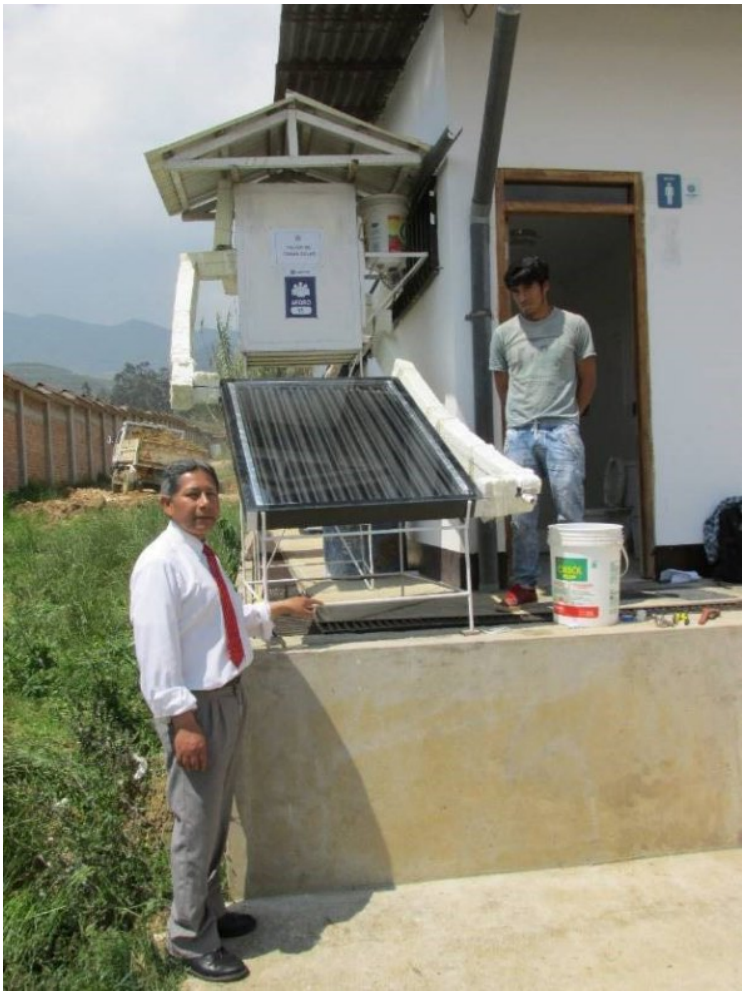

Figura 2. Terma solar con colector de tubos de $3 / 4$ " de PVC.

\section{Registro de datos de temperaturas}

Un tanque para almacenamiento de 100 litros de agua caliente aislado térmicamente se acopló al colector solar construido, que se ubicó sobre un soporte para posicionarlo en la dirección norte - sur, con el lado sur levantado $30^{\circ}$, para maximizar la captación de la radiación solar, siendo la trayectoria del sol de este a oeste, realizándose las conexiones hidráulicas de entrada y salida de agua.

Para la medición y registro de las temperaturas se emplearon cuatro sensores de temperatura DS18B20 con rango de $-55^{\circ} \mathrm{Ca} 125^{\circ} \mathrm{C}$ (Figura 3 ):

- Un sensor en la parte inferior derecha del colector solar, en la tubería por donde ingresará el agua fría (sensor de temperatura en la entrada del agua fría).

- El segundo en el interior del colector, ubicado en el centro de la parrilla de tubos de PVC del colector solar (sensor de temperatura en el interior del colector solar).

- El tercer sensor en la parte superior izquierda del colector, en la tubería por donde saldrá el agua caliente del colector solar (sensor de temperatura en la salida de agua caliente).
- El cuarto sensor en la parte interior del tanque de almacenamiento de agua caliente a una profundidad de $20 \mathrm{~cm}$ del nivel de agua. El sensor se colocó en una boya para que gradualmente vaya subiendo a medida que se va llenando el tanque con el agua, quedando siempre el sensor a una profundidad de $20 \mathrm{~cm}$, cuando el tanque se haya llenado completamente con agua.

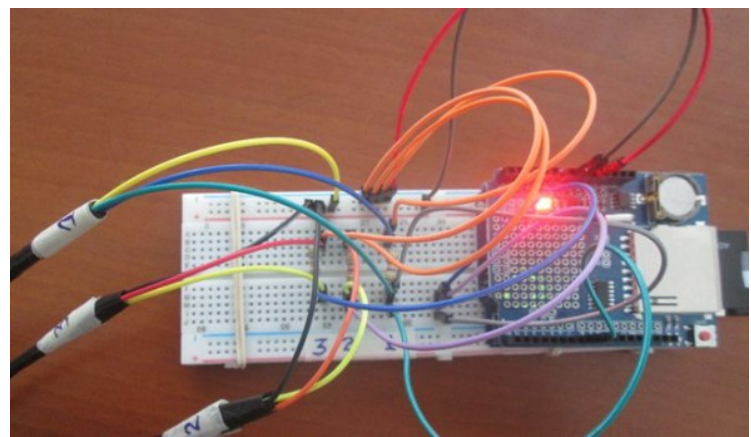

Figura 3. Montaje de Arduino UNO, Datalogger y sensores.

Como interface para la medición y registro automático de datos por 24 horas continuas, se usó el módulo Arduino UNO (Figura 3), almacenándose los datos en una memoria SD de $16 \mathrm{~Gb}$. Se programó el módulo Arduino UNO para el registro de temperaturas de los cuatro sensores en intervalos de 15 minutos. Cada día, a las 7:00 am se abrió la válvula del colector solar para el vaciado total del agua almacenada del día anterior, para luego llenar la terma solar con agua de la red pública, para la evaluación correspondiente. A medida que aumentó la incidencia de la radiación solar sobre la parrilla de tubos de PVC del colector durante el día, el agua caliente por efecto termosifón se fue almacenando gradualmente en la parte superior del tanque, $y$ de la base de este fluía el agua fría hacia el colector solar. Cada semana se descargaron los datos de temperaturas del Arduino UNO, en una computadora personal para ser procesados.

\section{Estación meteorológica del INDES-CES}

De la estación meteorológica (Davis, modelo Vantage Pro 2 Plus) localizada dentro del Campus de la Universidad Nacional Toribio Rodríguez de Mendoza de Amazonas, se obtuvieron datos desde el 15 de octubre al 15 de noviembre del 2017, totalizando 31 días de 
medición y registro de los siguientes datos:

- Radiación solar $\left(\mathrm{kW} / \mathrm{m}^{2}\right)$

- Precipitación (mm)

- Temperatura ambiente $\left({ }^{\circ} \mathrm{C}\right)$

- Velocidad del viento $(\mathrm{m} / \mathrm{s})$

\section{Registro de datos}

El registro de datos se realizó de dos modos:

- En el modo continuo de 24 horas, para evaluar el comportamiento de la conservación de la temperatura del agua en el tanque de almacenamiento de agua caliente.

- En el modo de ocho horas, horas solar pico (desde las 8:00 am - 4:00 pm), para evaluar el comportamiento del colector solar con la incidencia de la radiación solar durante las horas de luz del día para calentar el agua.

\section{Eficiencia del colector solar}

Según Valera (2007), la eficiencia de un colector $(\eta E)$, es la relación entre la energía térmica útil ganada por el colector y la energía solar recibida o disponible ese día:

$$
n E=\frac{\text { Energía térmica útil }(E t)}{\text { Energía solar recibida }(E T)}
$$

Energía térmica útil ganada: Et

$$
E t=m \times c \times \Delta T
$$

Donde:

$\mathrm{m}$ : masa de agua en la terma $=(0,6 \mathrm{a} 1) \times 105 \mathrm{~g}$

c: calor específico del agua $=4,18 \mathrm{~J} / \mathrm{g} \times{ }^{\circ} \mathrm{C}$

$\Delta \mathrm{T}=$ Temperatura máxima del agua $(4: 00 \mathrm{pm})-\mathrm{Tem}-$ peratura del agua en el tanque al inicio (8:00 am), ${ }^{\circ} \mathrm{C}$

Energía solar recibida: Es

$$
E T=e s \times A
$$

Donde:

es: área bajo la curva de la radiación solar vs tiempo

A: área del colector solar $=1 \mathrm{~m}^{2}$

\section{RESULTADOS}

La Tabla 1 corresponde al registro de datos desde las 8:00 hasta las 16:00 horas, lo que permitirá evaluar la eficiencia del colector solar de tubos de PVC. En esta se pueden observar los principales parámetros: el comportamiento de la temperatura ambiente, los valores de la radiación solar y los períodos del día.

Tabla 1. Comportamiento de la radiación solar

\begin{tabular}{ccccc}
\hline Hora & $\begin{array}{c}\text { Temperatura } \\
\text { ambiente }\left({ }^{\circ} \mathbf{C}\right)\end{array}$ & $\begin{array}{c}\text { Radiación } \\
\text { solar }\left(\mathbf{W} / \mathbf{m}^{2}\right)\end{array}$ & $\begin{array}{c}\text { Radiación } \\
\text { solar }\left(\mathbf{K W} / \mathbf{m}^{2}\right)\end{array}$ & Períodos del día \\
\hline 08:00 a.m. & 13,9 & 207 & 0,21 & Nublado \\
08:16 a.m. & 14,0 & 450 & 0,45 & Nublado \\
08:29 a.m. & 14,2 & 371 & 0,37 & Nublado \\
08:45 a.m. & 14,9 & 355 & 0,36 & Nublado \\
09:01 a.m. & 15,6 & 550 & 0,55 & Nublado \\
09:17 a.m. & 16,8 & 663 & 0,66 & Combinado \\
09:29 a.m. & 16,3 & 578 & 0,58 & Nublado \\
09:46 a.m. & 16,2 & 464 & 0,46 & Nublado \\
10:02 a.m. & 16,6 & 529 & 0,53 & Nublado \\
10:14 a.m. & 17,3 & 668 & 0,67 & Combinado \\
10:30 a.m. & 18,6 & 329 & 0,33 & Nublado \\
10:47 a.m. & 18,8 & 420 & 0,42 & Nublado \\
10:59 a.m. & 19,5 & 649 & 0,65 & Combinado \\
11:15 a.m. & 19,8 & 378 & 0,38 & Nublado \\
\hline
\end{tabular}


Tabla 1. Comportamiento de la radiación solar(Continuación).

\begin{tabular}{ccccc}
\hline Hora & $\begin{array}{c}\text { Temperatura } \\
\left.\text { ambiente }^{\circ} \mathbf{C}\right)\end{array}$ & $\begin{array}{c}\text { Radiación } \\
\text { solar }\left(\mathbf{W} / \mathbf{m}^{\mathbf{2}}\right)\end{array}$ & $\begin{array}{c}\text { Radiación } \\
\text { solar }\left(\mathbf{K W} / \mathbf{m}^{2}\right)\end{array}$ & Períodos del día \\
\hline 11:31 a.m. & 20,0 & 316 & 0,32 & Nublado \\
11:44 a.m. & 20,7 & 327 & 0,33 & Nublado \\
12:00 p.m. & 21,0 & 371 & 0,37 & Nublado \\
12:16 p.m. & 21,2 & 380 & 0,38 & Nublado \\
12:32 p.m. & 21,8 & 506 & 0,51 & Nublado \\
12:45 p.m. & 22,1 & 1315 & 1,32 & Soleado \\
01:01 p.m. & 21,2 & 1155 & 1,16 & Soleado \\
01:17 p.m. & 20,8 & 1088 & 1,09 & Soleado \\
01:29 p.m. & 21,1 & 1083 & 1,08 & Soleado \\
01:45 p.m. & 22,2 & 998 & 1,00 & Soleado \\
02:02 p.m. & 22,8 & 921 & 0,92 & Soleado \\
02:14 p.m. & 23,2 & 840 & 0,84 & Soleado \\
02:30 p.m. & 22,6 & 819 & 0,82 & Soleado \\
02:46 p.m. & 22,7 & 809 & 0,81 & Soleado \\
02:59 p.m. & 22,5 & 821 & 0,82 & Soleado \\
03:15 p.m. & 22,5 & 383 & 0,38 & Nublado \\
03:31 p.m. & 23,1 & 316 & 0,32 & Nublado \\
03:47 p.m. & 23,2 & 118 & 0,12 & Nublado \\
04:00 p.m. & 23,1 & 120 & 0,12 & Nublado \\
\hline
\end{tabular}

En la Tabla 1, se puede observar que en un día cualquiera, se presentan períodos nublados, combinados y soleados. Las temperaturas en el interior del colector solar mayores a las del tanque de almacenamiento de agua caliente, permitirán que ocurra el termosifón natural (Tabla 2).

Tabla 2. Comportamiento de las temperaturas en el colector solar de tubos de PVC.

\begin{tabular}{ccccc}
\hline Hora & $\begin{array}{c}\text { Temp. Entrada Agua } \\
\text { Fría }\end{array}$ & $\begin{array}{c}\text { Temp. Colector } \\
\text { Solar }\end{array}$ & $\begin{array}{c}\text { Temp. Salida } \\
\text { Agua Fría }\end{array}$ & Temp. Interior Tanque \\
\hline 08:00 a.m. & 15,25 & 25,31 & 17,87 & 16,31 \\
08:16 a.m. & 15,25 & 30,50 & 22,69 & 19,25 \\
08:29 a.m. & 17,44 & 35,94 & 30,44 & 24,12 \\
08:45 a.m. & 28,75 & 39,00 & 37,94 & 29,00 \\
09:01 a.m. & 29,12 & 41,38 & 38,88 & 28,87 \\
09:17 a.m. & 29,81 & 40,75 & 38,00 & 28,94 \\
09:29 a.m. & 29,75 & 38,81 & 29,06 \\
09:46 a.m. & 30,69 & 46,81 & 36,81 & 29,25 \\
10:02 a.m. & 31,06 & 48,38 & 41,44 & 30,37 \\
10:14 a.m. & 31,25 & 53,75 & 42,38 & 32,25 \\
10:30 a.m. & 31,75 & 56,69 & 44,00 & 34,19 \\
10:47 a.m. & 32,63 & 56,19 & 45,31 & 35,56 \\
10:59 a.m. & 32,81 & 57,00 & 46,00 & 36,56 \\
11:15 a.m. & 32,88 & 46,81 & 45,69 & 37,56 \\
11:31 a.m. & 33,38 & 47,44 & 41,19 & 38,25 \\
11:44 a.m. & 33,81 & 53,69 & 42,38 & 38,13 \\
12:00 p.m. & 34,94 & 47,50 & 43,25 & 37,56 \\
\hline
\end{tabular}


Tabla 2. Comportamiento de las temperaturas en el colector solar de tubos de PVC (Continuación).

\begin{tabular}{ccccc}
\hline Hora & $\begin{array}{c}\text { Temp. Entrada Agua } \\
\text { Fría }\end{array}$ & $\begin{array}{c}\text { Temp. Colector } \\
\text { Solar }\end{array}$ & $\begin{array}{c}\text { Temp. Salida } \\
\text { Agua Fría }\end{array}$ & Temp. Interior Tanque \\
\hline 12:16 p.m. & 35,88 & 47,44 & 43,94 & 35,69 \\
12:32 p.m. & 36,50 & 47,38 & 44,00 & 35,56 \\
12:45 p.m. & 37,25 & 45,00 & 43,13 & 35,44 \\
01:01 p.m. & 36,88 & 46,69 & 42,94 & 35,31 \\
01:17 p.m. & 37,50 & 46,75 & 43,88 & 35,25 \\
01:29 p.m. & 37,63 & 46,69 & 43,69 & 35,13 \\
01:45 p.m. & 38,13 & 45,13 & 43,06 & 35,06 \\
02:02 p.m. & 36,81 & 41,13 & 41,13 & 35,06 \\
02:14 p.m. & 35,81 & 39,63 & 39,75 & 35,00 \\
02:30 p.m. & 33,63 & 37,69 & 38,13 & 34,94 \\
02:46 p.m. & 31,25 & 35,63 & 36,19 & 34,81 \\
02:59 p.m. & 30,00 & 34,31 & 35,63 & 34,75 \\
03:15 p.m. & 28,19 & 33,56 & 33,88 & 34,75 \\
03:31 p.m. & 27,50 & 34,19 & 33,63 & 34,69 \\
03:47 p.m. & 26,50 & 33,25 & 32,63 & 34,63 \\
04:00 p.m. & 26,06 & 32,56 & 31,75 & 34,63 \\
\hline
\end{tabular}

\section{Eficiencia del colector solar en periodos nublados}

El promedio de la eficiencia obtenida para los días con periodos nublados fue: $\eta=0,3564$ o 35,64\% (Figura 4). El promedio de almacenamiento de agua caliente en el tanque fue: 87,10 litros.
- El día con mayor eficiencia alcanzada fue el día 20 $\operatorname{con} \eta=0,5675$ o $56,75 \%$.

- El día con menor eficiencia alcanzada fue el día 6 con $\eta=0,2754$ o $27,54 \%$.

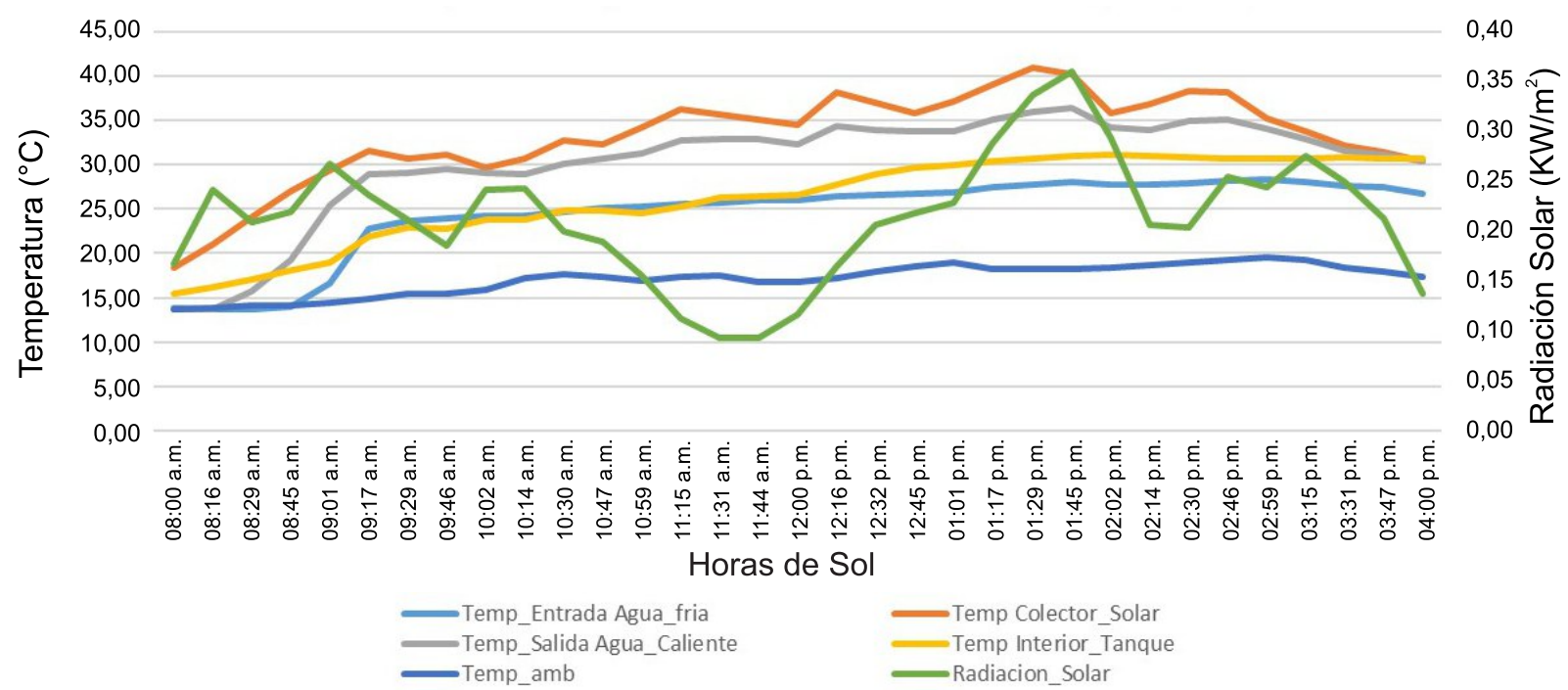

Figura 4. Comportamiento de las temperaturas durante 8 horas (8:00-16:00 horas) en periodos nublados.

Eficiencia del colector solar en periodos combinados

El promedio de la eficiencia obtenida para los días con periodos combinados fue: $\eta=0,3851$ o $38,51 \%$ (Figura 5). El promedio de almacenamiento de agua caliente en el tanque fue: 89,52 litros.

- El día con mayor eficiencia alcanzada fue el día 5 $\operatorname{con} \eta=0,5245$ o $52,45 \%$.

- El día con menor eficiencia alcanzada fue el día 28 $\operatorname{con} \eta=0,2625$ o $26,25 \%$. 


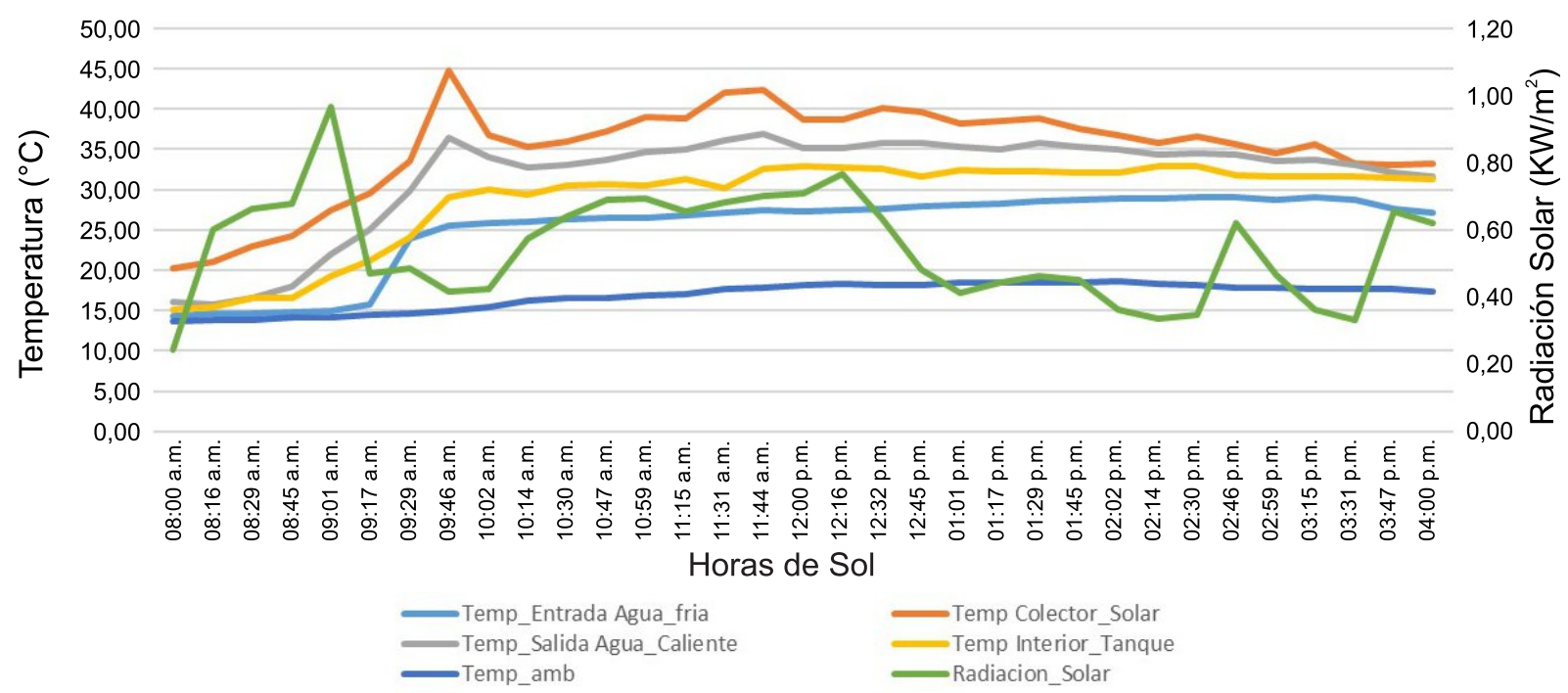

Figura 5. Comportamiento de las temperaturas durante 8 horas (8:00 - 16:00 horas), en periodos combinados.

\section{Eficiencia del colector solar en periodos soleados}

El promedio de la eficiencia obtenida para los días con periodos nublados fue: $\eta=0,4457$ o 44,57\% (Figura 6).

- El promedio de almacenamiento de agua caliente en el tanque fue: 90,51 litros.
- El día con mayor eficiencia alcanzada fue el día 20 con $\eta=0,5473$ o $54,73 \%$.

- Los días con menor eficiencia alcanzada fueron los días 2 y 6 con $\eta=0,0928$ o $9,28 \%$ y $\eta=0,2635$ o $26,35 \%$.

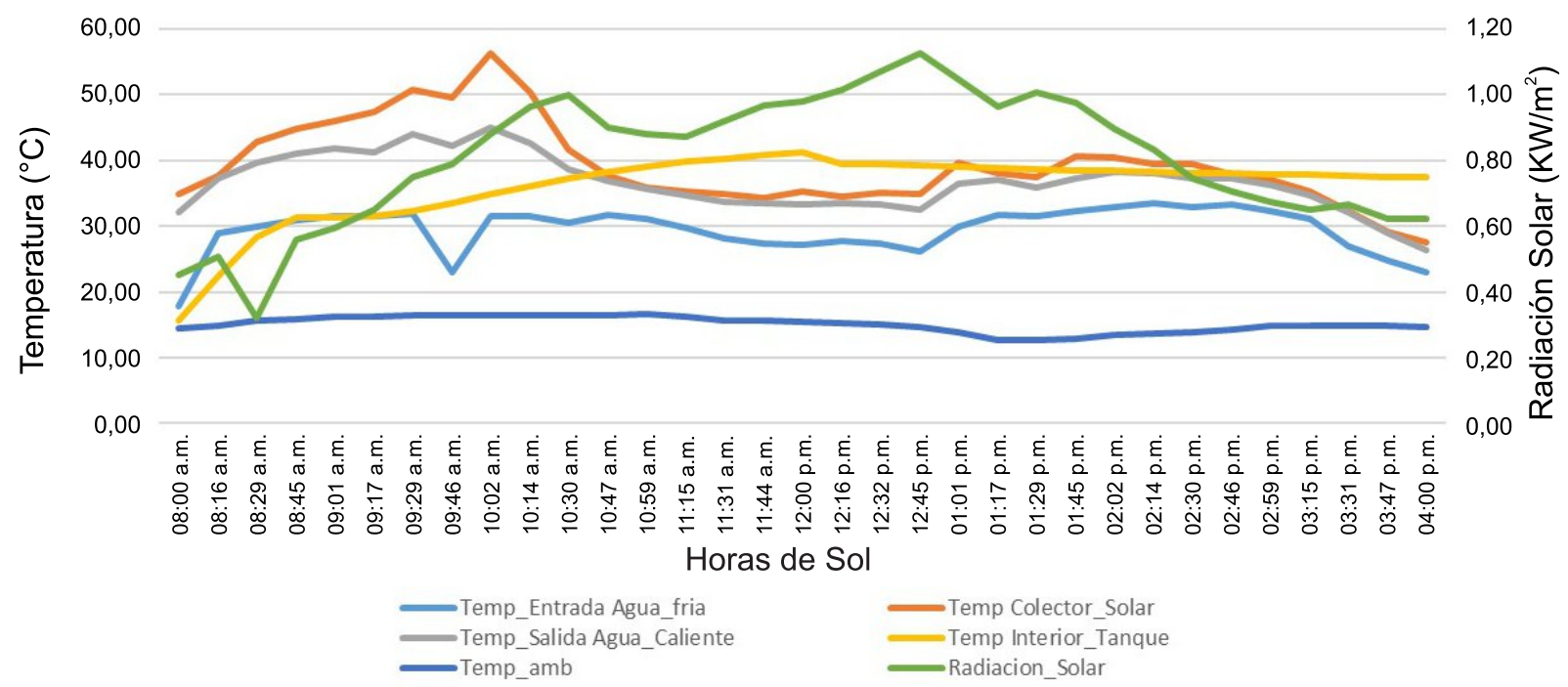

Figura 6. Comportamiento de las temperaturas durante 8 horas (8:00-16:00 horas), en periodos soleados.

\section{IV.DISCUSIÓN}

Del estudio realizado, se ha observado que, en los 31 días de evaluación, se presentaron cielos con tres niveles de nubosidad diaria, razón por la cual se categorizó un mismo día con periodos nublados, combinados y soleados. No fue posible catalogar un grupo de días, como totalmente nublados, combinados o soleados, por lo que la categorización de la nubosidad diaria conduce a que el colector solar capte distintos niveles de radiación solar.

Según lo expuesto por López et al. (2012), el factor de corrección de la radiación solar media diaria en función de la calificación de la nubosidad de un día específico, para la ciudad de Bogotá (Colombia), que tiene una altitud y condiciones climáticas similares a las de la ciudad de Chachapoyas (Amazonas), es de 0,50 para cielo nublado entre el 100 y $80 \%$ (o día lluvioso), y de 1,00 para cielo nublado entre 20 y $0 \%$ (día despejado), 
con valores intermedios en función del porcentaje de cielo nublado, lo cual tiene cierta subjetividad., 2012)

Este criterio guarda una similitud con el presente estudio realizado, dado que en un día específico se notó que presentó distintas categorías de nubosidad, lo que conllevó a clasificar como periodos de tiempo nublados, combinados y soleados. En la ciudad de Chachapoyas, en los 31 días de evaluación no se presentaron días netamente soleados o combinados, excepto dos días nublados. En el estudio realizado se clasificaron los periodos del día por el nivel de la radiación solar: nublado de 0 - 600 Watts, combinado de $600-800$ Watts y soleado mayor a 800 Watts, lo cual se puede medir con un equipo y así evitar subjetividades.

Referente a la eficiencia, en el presente estudio se utilizaron tubos de CPVC de $3 / 4$ de pulgada para un colector de $1 \mathrm{~m}^{2}$. La eficiencia alcanzada en promedio para días con periodos soleados fue de $\eta=0,4457$ o $44,57 \%$ para un tanque que almacenó 90 litros de agua a $38,25^{\circ} \mathrm{C}$, con una ganancia de temperatura de $21,94^{\circ} \mathrm{C}$, y con una radiación solar total del día de 4,50 $\mathrm{kW} \cdot \mathrm{h} / \mathrm{m}^{2}$.

Vigo y Trigoso (2012), evaluaron una terma solar de $100 \mathrm{~L}$ de agua en Chachapoyas, con un solo colector hecho de tubería y accesorios de CPVC de 1/2" y para este mismo tipo de día reportaron que la temperatura máxima del agua en el tanque de almacenamiento fue de $44^{\circ} \mathrm{C}$, una ganancia de temperatura de $24,5^{\circ} \mathrm{C}$, eficiencia de $52 \%$ con una radiación solar de 5,42 $\mathrm{kW} . \mathrm{h} / \mathrm{m}^{2}$. Por esto, se puede asegurar que el colector solar con tubos de CPVC de $1 / 2$ " es más eficiente que con tubos de $3 / 4$ de pulgada, debido a que la masa de agua contenida en los tubos de $1 / 2$ " es menor y el recorrido del calor del exterior del tubo al centro de la masa de agua que contiene es menor.

Lobato y Barrena (2016), evaluaron una terma solar con tanque de almacenamiento de agua de 100 litros y dos colectores de $1,5 \mathrm{~m}^{2}$ cada uno. Un colector tenía serpentín plano de tubo de cobre $1 / 2$ " y el otro tenía serpentín plano de tubo de CPVC de $1 / 2$ "; en la ciudad de Tarma (Región Junín, Perú). La radiación solar en día soleado superó los $1000 \mathrm{~W} / \mathrm{m}^{2}$, desde las 10:00 am hasta la 1:30 pm, debido a su clima seco por la escasa vegetación. Además manifestaron que el aumento y la disminución de la radiación solar tienen relación directa con la temperatura del interior de los colectores solares evaluados. Cuando la radiación solar superó $\operatorname{los} 0,8 \mathrm{~kW} / \mathrm{m}^{2}$, el colector hecho con tubos y accesorios de CPVC ganó más temperatura que el colector hecho con tubos y accesorios de cobre de iguales dimensiones, con una diferencia promedio de $20^{\circ} \mathrm{C}$.

Por lo tanto, existen estudios que permiten confirmar la hipótesis alternativa planteada, que establece la existencia de la relación directa entre la radiación solar y el tipo de días para la determinación de la eficiencia energética de colectores solares con tubos verticales de PVC en termas solares, Amazonas, 2017. Por ello, en un día despejado la temperatura interior del colector solar se elevará y la temperatura del agua tendrá el mismo efecto, caso contrario disminuirá, lo que indica una tendencia a un comportamiento directamente proporcional, es decir, que a medida que se incrementa la temperatura interior del colector solar, es porque se incrementa la radiación solar y viceversa. En conclusión,se puede afirmar que el efecto de la radiación solar es directamente proporcional sobre el calentamiento del agua.

Con el presente estudio realizado en la ciudad de Chachapoyas ( $2350 \mathrm{msnm}$ y $17^{\circ} \mathrm{C}$ de temperatura ambiente), con un colector solar de $1 \mathrm{~m}^{2}$ con tubos de CPVC de $3 / 4$ de pulgada y con un tanque de almacenamiento de 100 litros de agua, se han logrado los siguientes resultados: el promedio de la eficiencia obtenida para los días con periodos nublados fue $\eta=0,3564$ o $35,64 \%$, para los días con periodos combinados de $\eta=0,3851$ o $38,51 \%$ y para los días con periodos soleados de $\eta=0,4457$ o 44,57\%; con lo que se demuestra que brinda confort térmico aunque el día sea nublado.

Vigo y Trigoso (2012), en su evaluación de una terma solar con un colector de $1 \mathrm{~m}^{2}$ cuya parrilla de tubos verticales la hicieron de tubo de CPVC de 1/2" para agua caliente y accesorios del mismo material, y tanque de almacenamiento de agua de 100 litros aislado térmicamente; encontraron que la temperatura del 
agua en el tanque de almacenamiento a las 4:00 pm y la eficiencia en días soleados fue de $44^{\circ} \mathrm{C}$ y $52 \%$, para días combinados fue de $36^{\circ} \mathrm{C}$ y $48 \%$ y para días nubla$\operatorname{dos} 25^{\circ} \mathrm{C}$ y $30 \%$; mientras que la temperatura interior del colector fue de 96,63 y $38^{\circ} \mathrm{C}$, respectivamente. Por lo que se puede apreciar que el colector hecho con tubos y accesorios de PVC de $3 / 4$ " de la presente investigación tiene una eficiencia menor en 5 puntos porcentuales que la eficiencia del colector solar hecho por Vigo y Trigoso (2012) con tubos y accesorios de PVC de $1 / 2$ ", evaluados en las mismas condiciones ambientales; con lo que también se verifica que la eficiencia del colector solar es incrementada por el menor diámetro de sus tubos.

Según la UNAM (2010), el costo del calentador solar con tubos de cobre es de $\$ 1949$. Si lo comparamos con los calentadores a gas más económicos, los cuales tienen precios que oscilan entre los $\$ 1400$, y si tenemos en cuenta que los calentadores a gas además de la inversión inicial, se tiene que pagar el gas que se consume, que para el calentamiento de agua es de aproximadamente $\$ 100$ al mes, a los seis meses de uso ya se habrá recuperado la inversión que se tendría que hacer para construir el calentador solar. Por lo que se concluye que el calentador solar presenta realmente un beneficio económico, pues sólo requiere inversión inicial y su mantenimiento implica únicamente la limpieza de la cobertura de vidrio del colector solar, siendo su durabilidad de aproximadamente 15 años.

En el presente estudio, el colector solar de CPVC con tubos de $3 / 4$ pulgada y marco de aluminio tiene un costo global de 720 soles, la estructura de metal para soporte del colector solar y del tanque de almacenamiento de agua caliente, tuberías de conexión del colector con el tanque de almacenamiento de 100 litros de agua, tienen un valor de 460 soles, y el sistema de almacenamiento que incluye el tanque, caseta de madera para el tanque y el aislamiento térmico tienen un valor de 340 soles. En total la terma solar tiene un valor de 1520 soles, que no incluye mano de obra ni equipos de medición, y con esta terma no se tendrá que pagar la energía solar que emplea.
Para establecer el beneficio económico que genera, la comparamos con otra terma eléctrica de 110 litros que consume 2000 Watts, cuyo costo es de S/ 1500, y un gasto mensual adicional de S/ 120por consumo de energía eléctrica. Entonces queda demostrado que la terma solar es más económica, y con el ahorro de no pagar la energía eléctrica se puede financiar o recuperar la inversión en una terma solar.

Además, se debe destacar el beneficio ambiental de la terma solar que emplea una fuente de energía renovable gratuita y amigable con el medio ambiente, pues durante su construcción y funcionamiento no emite gases tóxicos ni de efecto invernadero.

\section{CONCLUSIONES}

Existe relación directa entre la radiación solar y el tipo de día para determinar la eficiencia energética de colectores solares con tubos verticales de PVC para una terma solar.

La incidencia de la radiación solar sobre el colector de PVC mantiene relación directa con la nubosidad del día, es decir, si los periodos del día son nublados, combinados o soleados, esto afectará:

- La eficiencia del colector solar de PVC.

- La temperatura del agua caliente almacenada en el tanque.

- El volumen de agua caliente almacenada en el tanque.

La radiación solar captada por el colector solar de PVC de $1 \mathrm{~m}^{2}$, en promedio para días con periodos:

- Nublados fue $1,90 \mathrm{KWh} / \mathrm{m}^{2}$ del total de 4,50 $\mathrm{KWh} / \mathrm{m}^{2}$

- Combinados fue $0,89 \mathrm{KWh} / \mathrm{m}^{2}$ del total de 4,50 $\mathrm{KWh} / \mathrm{m}^{2}$

- Soleados fue $2,04 \mathrm{KWh} / \mathrm{m}^{2}$ del total de 4,83 $\mathrm{KWh} / \mathrm{m}^{2}$

La eficiencia del colector solar en promedio para los días con periodos:

- Nublados fue $\eta=0,3564$ о 38,64\%.

- Combinados fue $\eta=0,3851$ o $38,51 \%$.

- Soleados fue $\eta=0,4457$ o $44,57 \%$.

La capacidad de almacenamiento de agua caliente en 
el tanque fue prefijada en 100 litros, sin embargo, al finalizar el día en los periodos:

- Nublados se logró almacenar en promedio 87,10 litros de agua caliente.

- Combinados se logró almacenar en promedio 89,52 litros de agua caliente.

- Soleados se logró almacenar en promedio 90,51 litros de agua caliente.

La radiación solar influye directamente sobre la temperatura interna del colector solar, produciendo como consecuencia el efecto termosifón que impulsa un determinado caudal de agua caliente del colector hacia el tanque de almacenamiento de agua caliente.

\section{REFERENCIAS BIBLIOGRÁFICAS}

Enperu. 2015 . Clima de Amazonas. http://www.enperu.org/informacion-utilclima-en-amazonas-ubicaci\%C3\%B3n-deamazonas-temperatura-amazonia.html (Consultada el 19 de mayo de 2017)

Estrada-Cajigal, V. y R. Almanza. 2005. Irradiaciones global directa y difusa en superficies horizontales e inclinadas, así como irradiación directa normal en la República Mexicana. Ciudad de México (México): Instituto de Ingeniería de la Universidad Nacional Autónoma de México.

Fasulo, A., J. Follari y J. Barral. 2007. Un Nuevo Colector Solar Acumulador. http://textoscientificos.com/energia/solar/nue vo-colector-solar-acumulador (Consultada el 12 de octubre de 2017)

Horn, M. 2006. “Los retos energéticos del Perú. El estado actual del uso de la energía solar en el Perú”. Perú Económico 29(11): 10-11.

INENCO (Instituto De Investigaciones En Energía No Convencional). 2007. UNSA CONICET. h t t p s : / / w w w. in ti.gob.ar/e r

e n O$\mathrm{va} / \mathrm{pdf} /$ colectores_solares_aguacaliente.pdf (Consultada el 17 de noviembre de 2017)

Lobato, G., y M. A. Barrena. 2016. "Eficiencia de una terma solar de cobre y colector de CPVC para zonas altoandinas." En XXII Simposio Peruano De Energía Solar. Huancayo (Perú).

López-Martínez, G. A., J. A. Mantilla-López y B. H. Poveda-Suarez. 2012. "Evaluación de un calentador solar de agua fabricado con tuberia PVC en serie y envases PET translúcidos." TECNURA 16: 120-128.

Paz Gutiérrez, J. C. 2006. Colectores Solares Planos. Tratamiento teórico Vol. I. Ciudad de Juárez (México): Instituto de Ingeniería y Tecnología de la Universidad Autónoma de Ciudad de Juárez.

Pilatowsky, I. 2005. Sistema de calentamiento solar de agua. Ciudad de México (México): Grupo Grabado de Morelos S.A.

UNAM (Universidad Nacional Autónoma de México). 2010. "Diseño y Construcción de un Calentador Solar" En XVIII Concurso Universitario Feria de las Ciencias. Ciudad de México (México)

Valera, P. A. 2007. Energía Solar II. Asamblea Nacional de Rectores (ANR). Lima (Perú).

Vigo, L. V. y R. R. Trigoso. 2012. Eficiencia térmica de un calentador solar de agua de 80 litros con colector de $1 \mathrm{~m}^{2}$. Tesis de Grado. Universidad Nacional Toribio Rodriguez de Mendoza de Amazonas. Chachapoyas (Perú).

Yadav, A. y V. K. Bajpai. 2011. "An experimental Study on Evacuated Tube Solar Collector for Heating of air in India." World Academy of Science Engineering and Technology 5(17): 725. 